\title{
A Novel Synthesis of Ketones Using 4,6-Pyrimidyl Diesters
}

\author{
Jae In Lee \\ Deparment of Chemistry Plant Resources Research Institute. College of Vatural Science, Duksung Women's Wniversitv, \\ Seoul 132-717. Korea. E-mail: jileeiáduksungack \\ Received Januarv 28. 2007
}

Key Words : 4.6-Pỵrimidỵl diesters, Acylating agents, Chelates. Substitution

The acylation of organometallics with carboxylic acid derivatives has been generally adopted as a chemoselective method of ketones fomation. ' Several types of acylating agents have been developed to avoid overaddition of organometallics to the desired ketones. suppressing by-products such as tertiary alcohols. The reaction of active esters having 2-pyridyl ligands such as $S$-(2-pyridyl)thioates:- 2-pyridyl esters, ${ }^{3}$ and $N$-methyl- $N$-(2-pyridyl)amides ${ }^{4}$ with Grignard reagents affords 6-membered chelates. which are dissociated to form the ketones by acidic hydrolysis. The treatment of $2-$ acy loxy -3-methylpy razines ${ }^{5}$ and 3-( $N$-acyl- $N$-methy lamino)1-methỵlimidazolium iodides ${ }^{6}$ with organolithiums or Grignard reagents also gave ketones but 2 equiv of organometallics were required. Among acylating agents to prepare ketones. $N$-methoxy- $N$-methylamides (Weinreb amides) ${ }^{7}$ have been widely used because they react with both organolithiums and Grignard reagents to fom various ketones including ynones and $\beta$-diketones via 5 -membered chelates without forming side products.

The synthesis of ketones has been accomplished by the reaction of carboxamides or trifluoro esters with organometallics. $N . N$-Dimethylacetamides. ${ }^{8} N$-acylaziridines. ${ }^{9}$ and ethyl trifluoroacetate ${ }^{10}$ reacted cleanly with both organolithiums and Grignard reagents to form the ketones upon acidic workup of the tetrahedral intermediates. where no significant amounts of overaddition products were not detected. The reaction of benzimidazole methiodide ${ }^{l]}$ and benzoyl cyanides ${ }^{12}$ with Grignard reagents also afforded the methyl ketones and diaryl ketones. respectively. Alternatively ketones were prepared from the nucleophilic addition of Grignard reagents to nitriles ${ }^{13}$ and $N$-(ethoxymethỵlene)- aniline. ${ }^{14}$ but the former proceeds in high temperature and the latter requires the additional step of dehydrogenation.

Although several groups have been employed as selective acylating agents. only one acyl group in the acylating agents has been transferred. There is no report on the development of acylating agents which produce 2 equiv of ketones from one acylating agent with Grignard reagents. In this paper we wish to report that 2 equiv of ketones can be efficiently prepared from 4.6-pydimidỵl diesters and Grignard reagents in high yields.

4.6-Pyrimidyl diesters 1 were readily prepared by the addition of 2 equiv of acid chlorides 3 to a mixture solution of 1 equiv of 4.6-dihydroxypyrimidine 2 and 2 equiv of triethylamine in methylene chloride at room temperature (Scheme 1). This reaction proceeded slowly because 2 was slightly soluble in methylene chloride. After completion of the reaction. the mixture was separated by aqueous workup and pure 1 was obtained after recrystallization from $20 \%$ EtOAc/n-hexane. However 4.6-pyrimidyl dipivalate was partially decomposed during aqueous workup and it was separated by dissolving in THF after evaporation of methỵlene chloride. followed by filtering off triethylamine hỵdrochloride in $92 \%$ yield.

The successful synthesis of ketones 4 using 1 was accomplished by the reaction with Grignard reagents in THF at 0 ${ }^{\circ} \mathrm{C}$. It was detenmined that 2 equiv of Grignard reagents converted 1 to the corresponding ketones without concomitant addition to the products. For example, the addition of 2 equiv of isobutylmagnesium chloride to a solution of 1 equiv of 4,6-pyrimidyl dibenzoate in $\mathrm{THF}$ at $0^{\circ} \mathrm{C}$ led to the fomation of the precipitate. which was hydrolyzed with<smiles>Oc1cc(O)ncn1</smiles>

2<smiles>[R]C(=O)Cl</smiles>

3

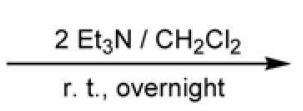

$\mathrm{R}=\mathrm{C}_{6} \mathrm{H}_{5}, 90 \% ; \mathrm{o}-\mathrm{Cl}-\mathrm{C}_{6} \mathrm{H}_{4}, 87 \% ; t-\mathrm{Bu}, 92 \%$ $m-\mathrm{Cl}-\mathrm{C}_{6} \mathrm{H}_{4}, 89 \% ; p-\mathrm{MeO}-\mathrm{C}_{6} \mathrm{H}_{4}, 93 \%$<smiles>[R]C(=O)Oc1cc(OC([R])=O)ncn1</smiles>

1

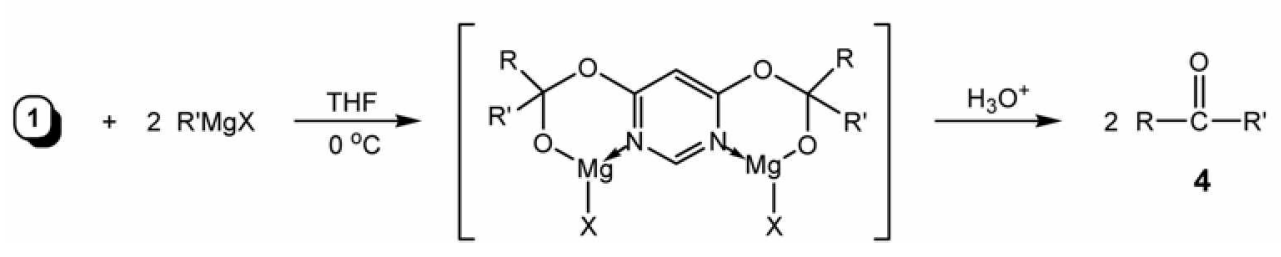

Scheme 1 
saturated $\mathrm{NH}_{4} \mathrm{Cl}$ solution to give 3 -methylbutyrophenone in $92 \%$ yield. There was no observable side product such as the corresponding tertiary alcohol due to the subsequent nucleophilic addition of Grignard reagent to the desired ketone. The success of this acylation seemed to result from the formation of the 6-mentbered chelate between magnesium cation of Grignard reagent and carbonyl oxygen/ring nitrogen of 1 , which was dissociated to give 4 after acidic hydrolysis.

As shown in Table 1, various ketones were efficiently synthesized by this method in ligh yields (76-93\%). The reaction worked well with both aliphatic and aromatic Grignard reagents. Electron withdrawing (te-th) and electron donating group (4i. $+\mathbf{j})$ in 4,6-pyrimidyl dibenzoate didn't influence on the nucleophilic acyl substitution of 1 by Grignard reagents. Significantly. the reactions are not limited to primary and secondary Grignard reagents. Thus. the reaction of 4,6-pyrimidyl dipivalate with 2-mesity Imagnesium bromide afforded the sterically hindered 2, 4',6'-trimethyl2.2-dimethylpropiophenone (4m) in $84 \%$ yield. However,

Table 1. Preparation of ketones from 4,6-pyrimidyl diesters and Grignard reagents ${ }^{\prime t}$

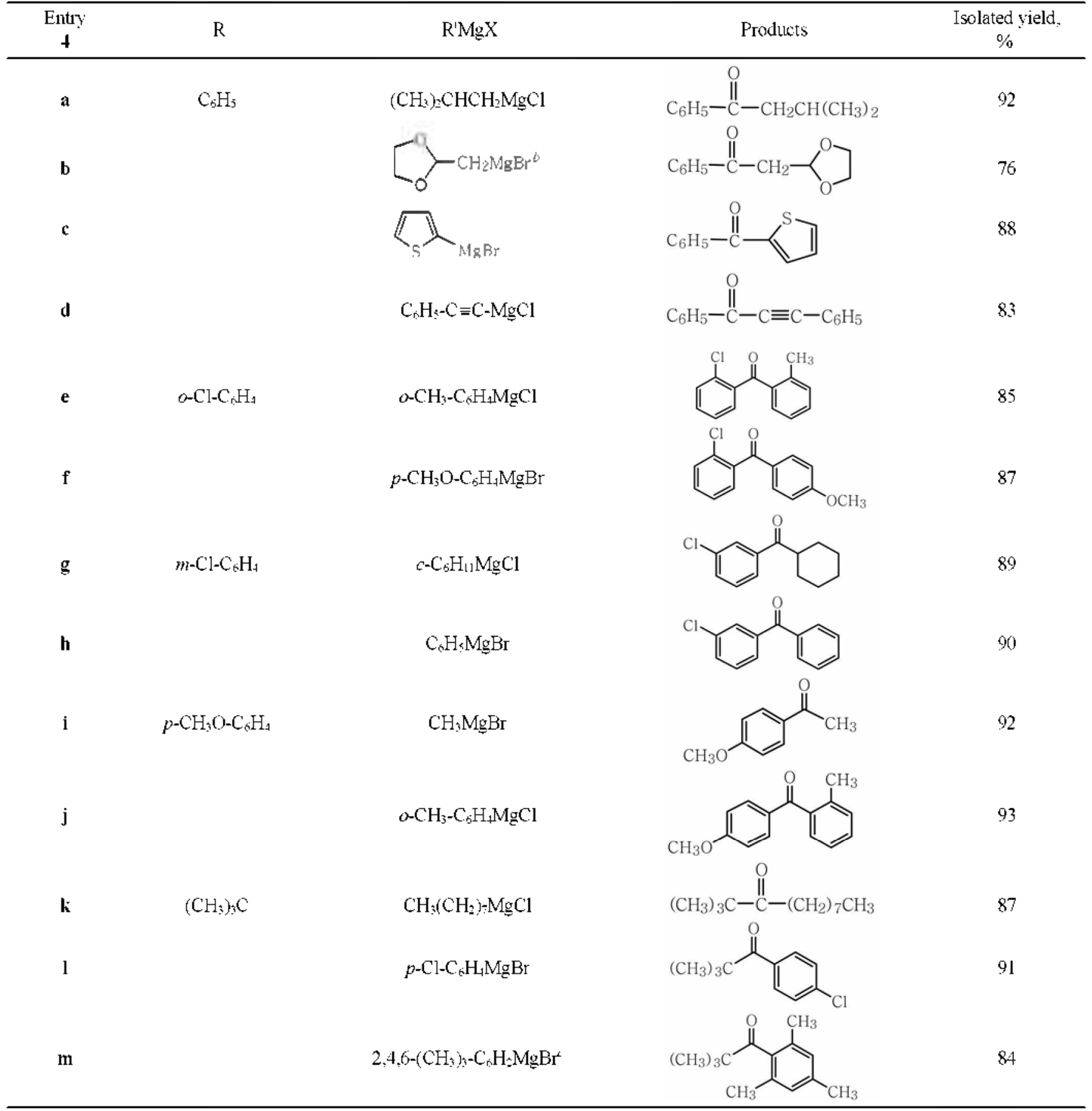

"The reaction was carried out at $0^{\circ} \mathrm{C}$ for $10^{\circ}$ min. "The reaction was carried out at room temperature for $1.5 \mathrm{~h}$. "The reaction was carried out between 0 " $\mathrm{C}$ and room temperature for $0.5 \mathrm{~h}$. 
the reaction of 4.6-pyrinidyl dibenzoate with (1,3-dioxolan2-ylnethyl)magnesium bromide was completed in $1.5 \mathrm{~h}$ at room temperature to give $\alpha$-(1.3-dioxolan-2-yl)acetophenone $(+b)$ in $76 \%$ yield. reflecting on the decreased nucleophilicity.

In conclusion the present method provides a novel synthesis of various ketones from 1 and Grignard reagents. It offers advantages: (i) the reactions are clean, (ii) the reaction times are short. and (iii) 2 equiv of ketones is synthesized from 1 equiv of 1 in high yields; thus may be utilized in many synthetic applications.

\section{Experimental Section}

Preparation of 4,6-pyrimidyl dibenzoate (General procedure). To a solution of 4.6-dihydroxypyrimidine (561 $\mathrm{mg}$. $5.0 \mathrm{mmol})$ and triethylamine $(1.46 \mathrm{~mL}, 10.5 \mathrm{mmol})$ in methylene chloride $(40 \mathrm{~mL})$ was added benzoyl chloride $(1.4 \mathrm{~g} .10 .0 \mathrm{~nm}$ ol) at room temperature. After being stirred overnight the mixture was poured into saturated $\mathrm{NaHCO}_{3}$ solution $(40 \mathrm{~mL})$. extracted with methylene chloride. and washed with brine $(40 \mathrm{~mL})$. The aqueous phase was reextracted twice with methylene chloride $(2 \times 25 \mathrm{~mL})$. The combined organic phases were dried over $\mathrm{MgSO}_{4}$. filtered. and concentrated in vicio. The residue was recrystallized fron $20 \% \mathrm{EtOAc} / n$-hexane to give 4,6 -pyrimidyl dibenzoate (1.44 g. 90\%): mp 97-98 ${ }^{\circ} \mathrm{C} ;{ }^{1} \mathrm{H}$ NMR (300 $\left.\mathrm{MHz}_{2} \mathrm{CDCl}_{3}\right)$ $\delta 8.98(\mathrm{~s}, 1 \mathrm{H}), 8.21-8.24(\mathrm{nl} .4 \mathrm{H}) .7 .65-7.72(\mathrm{~m}, 2 \mathrm{H}), 7.50-$ 7.57 (n. $4 \mathrm{H}), 7.4 \mathrm{l}(\mathrm{s}, \mathrm{IH}) ;{ }^{13} \mathrm{C} \mathrm{NMR}\left(75 \mathrm{MHz}, \mathrm{CDCl}_{3}\right)$ $\delta$ I67.1. 163.2. 159.0, 134.6, 130.6, 128.8, 128.0. 105.5; FTIR $(\mathrm{KBr}) 3062,1746(\mathrm{C}=\mathrm{O}) .1569 .1368,1243,1049,696$ $\mathrm{cnl}^{-1}$.

Preparation of 3-methylbutyrophenone 4a (General procedure). To a solution of 4.6-pyrinidyl dibenzoate (481 ing. $1.5 \mathrm{mmol})$ in THF (15 mL) was added isobutylnagnesium chloride $(0.5 \mathrm{M}$ in THF, $6.0 \mathrm{~mL}, 3.0 \mathrm{mumol})$ at $0^{\circ} \mathrm{C}$. After being stirred for $10 \mathrm{~min}$. the mixture was quenched with saturated $\mathrm{NH}_{4} \mathrm{Cl}$ solution $(3 \mathrm{~mL})$ and $\mathrm{THF}$ was evaporated in vacto. The mixture was poured into saturated $\mathrm{NH}_{4} \mathrm{Cl}$ solution $(30 \mathrm{~mL})$ and extracted with methylene chloride $(3 \times 25 \mathrm{~mL})$. The combined organic phases were dried over $\mathrm{MgSO}_{4}$. filtered. and concentrated in vacio. The residue was purified by vacuum distillation using Kugelrolur apparatus to give $4 \mathbf{a}\left(448 \mathrm{mg}\right.$. 92\%): ${ }^{\mathrm{l}} \mathrm{H}$ NMR $(300 \mathrm{MHz}$. $\left.\mathrm{CDCl}_{3}\right) \delta 7.93-7.97(\mathrm{~m}, 2 \mathrm{H}), 7.53-7.56(\mathrm{~m}, \mathrm{lH}), 7.43-7.48$ (m. $2 \mathrm{H}$ ), 2.83 (d. $J=6.9 \mathrm{~Hz}, 2 \mathrm{H}$ ). 2.30 (septet, $J=6.7 \mathrm{~Hz}$. 1H). $1.00(\mathrm{~d} J=6.7 \mathrm{~Hz}, 6 \mathrm{H}):{ }^{13} \mathrm{C}$ NMR $\left(75 \mathrm{MHz}^{\circ} \mathrm{CDCl}_{\hat{j}}\right) \delta$ 200.3. 137.4. 132.9, 128.5, 128.1. 47.5. 25.2. 22.8, FT-IR (film) 3061. 2957, 2870, I685 (C=O), 1598, 1448. 1406. $1385,1365,1284,1213,1006,752,691 \mathrm{~cm}^{-1}$; Ms mz (\%) $162\left(\mathrm{M}^{-}, 17\right), 120(30), 105(100) .77(43), 5 \mathrm{l}(\mathrm{ll})$.

$\alpha$-(1,3-Dioxolan-2-yl)acetophenone (4b): ${ }^{1} \mathrm{H}$ NMR (300 $\left.\mathrm{MHz} \mathrm{CDCl}_{\mathfrak{j}}\right) \delta 8.07(\mathrm{~d}, J=7.1 \mathrm{~Hz} .2 \mathrm{H}$ ). $7.53-7.59(\mathrm{~m} . \mathrm{lH})$. $7.41-7.46(\mathrm{~m} .2 \mathrm{H}), 6.52\left(\mathrm{dd}, J_{1}=14.3 \mathrm{~Hz}, J_{2}=6.8 \mathrm{~Hz}, \mathrm{IH}\right.$ ). $4.56(\mathrm{t} . J=4.7 \mathrm{~Hz}, 2 \mathrm{H}), 4.24\left(\mathrm{dd}, J_{1}=14.3 \mathrm{~Hz} . J_{2}=2.3 \mathrm{~Hz}\right.$. $1 \mathrm{H}), 4.07\left(\mathrm{dd}, J_{1}=6.8 \mathrm{~Hz}, J_{2}=2.3 \mathrm{~Hz} . \mathrm{HH}\right), 4.02(\mathrm{t} . J=4.7$ $\mathrm{Hz}, 2 \mathrm{H}):{ }^{13} \mathrm{C}$ NMR $\left(75 \mathrm{MHz} . \mathrm{CDCl}_{3}\right) \delta \mathrm{I} 66.5,15 \mathrm{l} .5 .133 .1$.
129.7, 128.4, 87.1. 65.9, 63.2; FT-IR (film) 3063. 2955. $2877.1722(\mathrm{C}=\mathrm{O}), 1618,1452,1366,1274,1111.823,711$ $\mathrm{cm}^{-1}$; Ms mz (\%) $192\left(\mathrm{M}^{-}, 1\right) .149$ (83). 105 (100), 77 (51).

Phenyl 2-thienyl ketone (4c): ${ }^{1} \mathrm{H}$ NMR $(300 \mathrm{MHz}$, $\left.\mathrm{CDCl}_{3}\right) \delta 7.88(\mathrm{~d}, J=8.0 \mathrm{~Hz}, 2 \mathrm{H}), 7.72(\mathrm{~d}, J=4.9 \mathrm{~Hz}, 1 \mathrm{H})$, 7.64 (d. $J=3.8 \mathrm{~Hz} . \mathrm{lH}), 7.56-7.6 \mathrm{l}(\mathrm{m}, \mathrm{lH}), 7.47-7.53(\mathrm{~m}$, 2H). 7.14-7.17 (m. 1H); ${ }^{13} \mathrm{C}$ NMR $\left(75 \mathrm{MHz} . \mathrm{CDCl}_{3}\right) \delta$ $188.2,143.6,138.1 .134 .9 .134 .2,132.3,129.2$. 128.4. 128.0: FT-IR (KBr) 3099, 1633 (C=O), 1597. 1411, 1286. 1052. $842.715 \mathrm{~cm}^{-1}$. Ms $m z(\%) 188\left(\mathrm{M}^{-} .100\right) .111(97)$. $105(33), 77(34)$.

1,3-Diphenyl-2-propyn-1-one (4d): ${ }^{1} \mathrm{H}$ NMR (300 MHz, $\mathrm{CDCl}_{3}$ ) $\delta 8.21-8.25$ (n. 2H). 7.67-7.70 (m, 2H), 7.63-7.67 (m, IH). 7.39-7.54 (m. 5H); ${ }^{13} \mathrm{C}$ NMR (75 MHz, CDCl $) \delta$ 178.0, 136.9, 134.1. 133.1. 130.8, 129.6, 128.7. 128.6. 120.1, 93.1. 86.9; FT-IR (film) 3061. 2198, 1637 (C=O). 1597. 1285. 1012, 757. $696 \mathrm{~cm}^{-1}$; Ms $m z(\%) 206\left(\mathrm{M}^{+} .6 \mathrm{l}\right)$, $178(100), 176(16), 129(68)$.

2-Chloro-2'-methylbenzophenone (4e): ${ }^{1} \mathrm{H}$ NMR $(300$ $\mathrm{MHz}, \mathrm{CDCl}_{3}$ ) $\delta 7.37-7.44$ (m. $4 \mathrm{H}$ ). $7.28-7.37$ (m. 3H). 7.15$7.22(\mathrm{~m}, \mathrm{lH}), 2.57(\mathrm{~s}, 3 \mathrm{H}):{ }^{12} \mathrm{C}$ NMR $\left(75 \mathrm{MHz}^{\left.-\mathrm{CDCl}_{3}\right)} \delta\right.$ 197.3, 139.6, 139.5. 136.9. 132.0, 131.9, 131.8. 131.5. $131.3,130.3 .129 .9 .126 .7,125.5,21.2$ : FT-IR (film) 3064 3021. 2965. 2926. 1671 (C=O), 1589, 1434, 1301. 1055. $926,743 \mathrm{~cm}^{-1}$. Ms $m z(\%) 232\left(\mathrm{M}^{+}+2,11\right), 231$ (13). 230 $\left(\mathrm{M}^{-}, 32\right) .229(39), 195(100) .141$ (7). $139(21) .119(28) .91$ (33).

2-Chloro- 4 -'methoxybenzophenone ( 4 f): ${ }^{1} \mathrm{H}$ NMR (300 $\left.\mathrm{MHz} \mathrm{CDCl}_{3}\right) \delta 7.79(\mathrm{~d} . J=7.0 \mathrm{~Hz}, 2 \mathrm{H}) .7 .40-7.47(\mathrm{~m}, 2 \mathrm{H})$, $7.32-7.40(\mathrm{~m} .2 \mathrm{H}) .6 .93(\mathrm{~d}, J=7.0 \mathrm{~Hz} .2 \mathrm{H}), 3.87(\mathrm{~s}, 3 \mathrm{H}):{ }^{13} \mathrm{C}$ NMR $\left(75 \mathrm{MHz}_{2} \mathrm{CDCl}_{3}\right) \delta 194.2,164.5,139.4,132.9,131.5$, $131.2,130.4 .129 .8 .129 .3,127.1,114.3 .55 .9$. FT-IR (KBr) 3057. 3013. 2973. $1657(\mathrm{C}=\mathrm{O}) .1598 .1433 .1255 .1150$. 1021. $862.762 \mathrm{~cm}^{-1}$; Ms $m z(\%) 248\left(\mathrm{M}^{+}+2,14\right), 246\left(\mathrm{M}^{-}\right.$. 4l). 139 (12). 135 (100), 77 (11).

3-Chlorophenyl cyclohexyl ketone (tg): ${ }^{\text {H }}$ NMR (300 $\left.\mathrm{MHz}_{3} \mathrm{CDCl}_{3}\right) \delta 7.88-7.90$ (n. $1 \mathrm{H}$ ). 7.81 (d. $J=7.7 \mathrm{~Hz}, \mathrm{lH}$ ), $7.50-7.53(\mathrm{~m} .1 \mathrm{H}) .7 .40\left(\mathrm{dd} . J_{1}=7.8 \mathrm{~Hz} . J_{2}=7.8 \mathrm{~Hz} . \mathrm{lH}\right)$, 3.15-3.24 (m, 1H). 1.70-1.92 (m,5H). 1.23-1.56 (m. 5H): ${ }^{13} \mathrm{C}$ NMR $\left(75 \mathrm{MHz} . \mathrm{CDCl}_{2}\right) \delta 202.9 .138 .4 .135 .3,133.0$. $130.3,128.7,126.7,46.1,29.7,26.3,26.1$ : FT-IR (film) 3067. 2931. 2854, 1681 (C=O), 1449. 1204.980.894.800. $730 \mathrm{~cm}^{-1}$. Ms $m z(\%) 224\left(\mathrm{M}^{+}+2.6\right) .222\left(\mathrm{M}^{-} .18\right) .187$ (35). 167 (19). 141 (36). 139 (100), $113(10) .111(30)$.

3-Chlorobenzophenone (4h): ${ }^{1} \mathrm{H}$ NMR $(300 \mathrm{MHz}$, $\mathrm{CDCl}_{3}$ ) $\delta 7.76-7.80$ (n. 3H), 7.39-7.77 (m. 6H): ${ }^{13} \mathrm{C}$ NMR $\left(75 \mathrm{MHz} \mathrm{CDCl}_{2}\right) \delta 195.2,139.3 .136 .9,134.6 .132 .8,132.3$. 130.0, 129.9. 129.6, 128.5. 128.1: FT-IR (KBr) 3064. 1662 $(\mathrm{C}=\mathrm{O}) .1568,1446,1284,1077,897,780,764,719,697$ $\mathrm{cm}^{-1}$; Ms $m z(\%) 218\left(\mathrm{M}^{-}+2.19\right), 216\left(\mathrm{M}^{+} .54\right), 141$ (10), $139(29), 113(7) .111(20), 105(100) .77(40)$.

4-Methoxyacetophenone (4i): ${ }^{1} \mathrm{H}$ NMR $(300 \mathrm{MHz}$, $\left.\mathrm{CDCl}_{3}\right) \delta 7.93(\mathrm{~d}, J=8.9 \mathrm{~Hz}, 2 \mathrm{H}), 6.93(\mathrm{~d}, J=8.9 \mathrm{~Hz}, 2 \mathrm{H})$, 3.87 (s. 3H), 2.55 (s. 3H); ${ }^{12} \mathrm{C}$ NMR (75 MHz, CDCl $) \delta$ $196.8,163.5,130.6,130.3,113.7,55.5,26.4$; FT-IR (film) $3004.2967,1674(\mathrm{C}=\mathrm{O}), 1601.1358,1258,1172,1027.834$ $\mathrm{cm}^{-1}$. Ms $m z(\%) 150\left(\mathrm{M}^{+} .32\right), 135$ (100). 107 (15). 92 
(16). $77(25)$.

2-Methyl-4'-methoxybenzophenone (4j): ${ }^{1} \mathrm{H}$ NMR (300 $\left.\mathrm{MHz} \mathrm{CDCl}_{3}\right) \delta 7.80(\mathrm{~d}, J=9.1 \mathrm{~Hz} .2 \mathrm{H}) .7 .35-7.40(\mathrm{~m} . \mathrm{lH})$. $7.21-7.35$ (m, 3H), 6.93 (d. $J=9.1 \mathrm{~Hz}, 2 \mathrm{H}$ ), 3.87 (s. $3 \mathrm{H}$ ). $2.30(\mathrm{~s}, 3 \mathrm{H}) ;{ }^{13} \mathrm{C}$ NMR $\left(75 \mathrm{MHz}, \mathrm{CDCl}_{3}\right) \delta$ 197.8. 164.1. 139.6. 136.6. 132.9, 131.2, 130.9. 130.2, 128.3, 125.6. 114.1. 55.9, 20.2: FT-IR (film) 3064. 3014, 2961, 1655 $(\mathrm{C}=\mathrm{O}), 1598.1458,1257.1149 .1028,846,750 \mathrm{~cm}^{-1}: \mathrm{Ms}$ $m z(\%) 226\left(\mathrm{M}^{-}, 33\right) .225(71), 195(100) .135(40), 91$ (19). $77(15)$

2,2-Dimethyl-3-undecanone (+k): ${ }^{1} \mathrm{H}$ NMR $(300 \mathrm{MHz}$. $\left.\mathrm{CDCl}_{3}\right) \delta 2.47$ (t. $\left.J=7.3 \mathrm{~Hz}, 2 \mathrm{H}\right), 1.50-1.60(\mathrm{~m} .2 \mathrm{H}), 1.23 \cdot$ $1.32(\mathrm{~m}, 10 \mathrm{H}) .1 .13(\mathrm{~s}, 9 \mathrm{H}) .0 .88(\mathrm{t}, J=6.8 \mathrm{~Hz} .3 \mathrm{H}):{ }^{13} \mathrm{C}$ NMR $\left(75 \mathrm{MHz} . \mathrm{CDCl}_{3}\right) \delta 216.2,44.1,36.5,31,9,29.5 .29 .4$. 29.2, 26.4, 24.0. 22.7. 14.1: FT-IR (film) 2957. 2926, 1707 $(\mathrm{C}=\mathrm{O}), 1465.1365 .1073,723 \mathrm{~cm}^{-1}$ : Ms $m z(\%) 198\left(\mathrm{M}^{+}\right.$. 3), $141(66), 71(38) .57(100)$.

4'-Chloro-2,2-dimethylpropiophenone (4l): ${ }^{1} \mathrm{H}$ NMR $\left(300 \mathrm{MHz} . \mathrm{CDCl}_{3}\right) \delta 7.67($ d. $J=7.7 \mathrm{~Hz}, 2 \mathrm{H}) .7 .38($ d. $J=$ $7.7 \mathrm{~Hz}, 2 \mathrm{H}), 1.34(\mathrm{~s}, 9 \mathrm{H}) ;{ }^{13} \mathrm{C}$ NMR $\left(75 \mathrm{MHz} . \mathrm{CDCl}_{3}\right) \delta$ 208.0. 137.6, 136.9. 129.9. 128.7, 44.5, 28.4; FT-IR (film) 3072. 2971, 2932, $1675(\mathrm{C}=\mathrm{O}) .1588,1476.1399 .1366$. 1189. 1091, 961, $839 \mathrm{~cm}^{-1}:$ Ms $m z(\%) 196\left(\mathrm{M}^{-}, 2\right), 1+1$ (37), $139(100)$. $111(20), 85$ (3). $57(15)$.

2',4',6'-Trimethyl-2,2-dimethylpropiophenone (4m): ${ }^{1} \mathrm{H}$ NMR $\left(300 \mathrm{MHz}\right.$. $\left.\mathrm{CDCl}_{3}\right) \delta 6.81$ (s. $2 \mathrm{H}$ ). 2.23 (s. 3H), 2.13 (s. $6 \mathrm{H}), 1.23(\mathrm{~s}, 9 \mathrm{H}):{ }^{13} \mathrm{C} \mathrm{NMR}\left(75 \mathrm{MHz}, \mathrm{CDCl}_{3}\right) \delta 219.8$. 140.0. 137.7, 132.4. 128.8, 45.2. 28.5. 21.3, 20.8: FT-IR (film) 2968. 2931. $1687(\mathrm{C}=\mathrm{O}), 1476,1378.1364 .1146$. $929,850 \mathrm{~cm}^{-1}$; Ms $m z(\%) 204\left(\mathrm{M}^{+} .1\right), \mathrm{l}+8(12) .147(100)$. $119(20), 91(9)$.

Acknowledgment. This work was partially supported by the Korea Research Foundation Grant funded by the Korea Govenmment (MOEHRD), Basic Research Promotion Fund
(KRF-2005-005-J13001) during the subbatical year of Duksung Women's University (2006).

\section{References}

1. (a) O'neill. B. T. In Comprehensine Organic Swmhesis: Trost. B M.. Ed.: Pergannon Press: Oxford. U. K.. 1991: Vol. 1. p 397. (b) Walter, D. S. In Comprehensive Organic Functional Group Transfomations: Katritzky, A. R., Ed:- Elsevier Science Ltd: Oxford, U. K.. 1995: Vol. 3. p 277. (c) Aldabbagh. F. In Comprehensive Organic Functional Group Transformations II: Katritzky. A. R.. Ed.: Elsevier Ltd.: Oxford. U. K.. 2005: Vol. 3. p 267.

2. (a) Mukaiyama. T.: Araki. M.: Takei. H. J. Am. Chem. Soc. 1973. 95. 4763. (b) Nicolaou, K. C.: Claremon, D. A.: Papahatjis, D. P. Tetrahedron Lett. 1981, 22. 4647. (c) Vazquez, J; Albericio. F. Tetrahedron Lett. 2002. 13.7499

3. Araki. M.: Sakata. S.: Takei. H.: Mukaiyama. T. Bull. Chen. Soc. Jpm. 1974. 17.1777 .

4. Meyers. A. I.: Comins. D. L. Tetrohedron Lett 1978. 19. 5179.

5. Abe. K.: Sato. T.; Nakamura, N.: Sakan, T. Chem Lett 1977.645.

6. (a) Heras. M. A.; Molina, A.; Vaquero, J. J.: Navio, J. L. G.; Alvarez-Builla. J. J. Org. Chem. 1993, 58. 5862 (b) Heras. M. A. vaquero. I. T.: Navio. J. L. G.: Alvarez-Builla. T. Tetrohedron 1996. 52.14297 .

7. (a) Nahm1. S.: Weinreb. S. M. Tetrahedron Lett. 1981. 22. 3815 (b) Sibi. M. P. Org. Prep. Proced Int. 1993, 25, 15. (c) Liu, J. Ikemoto, N.: Petrillo. D.: Ammstrong, J. D. Tetrahedron Lett 2002. 43. 8223 .

8. (a) Olah. G. A.: Prakash. G. K. S.: Arvanaghi. M. Swnhesis 1984. 228. (b) Comitns. D. L: Hong. H. J. An. Chem. Soc. 1991. 113. 6672 .

9. Wattanasin. S.: Kathawala. F. G. Tetrahedron Lett. 1984. 25.811.

10. Creary, X. J. Org. Chem $1987,52,5026$.

11. Yang. B. Q: Qiang. J. L.: Bai. Y. J. Lu, J.: Shi, Z. Chinese d. Chent 2003. 2l. 1210 .

12. Duplais. C.: Bures. F.: Sapountzis. I.: Korn. T. J.: Cahiez. G.: Knochel. P. Angew. Chem. Int Ed. 2004. 13.2968.

13. (a) Itsuno. S: Miyazaki, K.: Ito, K. Tetrohedron Lett. 1986. 27. 3033. (b) Weiberth, F. J.; Hall, S. S. J. Org. Chem 1987, 52. 3901.

14. Strekowski. L.; Wydra, R. L.: Cegla. M. T.: Czarny. A.: Patterson. S. J. Org. Chem. 1989. 5t.6120. 